\title{
Auftragskommunikation für die Kirche: Werbung, Öffentlichkeitsarbeit und Social Marketing
}

\author{
von Michael Krzeminski
}

Während Presse- und Öffentlichkeitsarbeit seit geraumer Zeit einen festen Platz im Spektrum kirchlicher Kommunikationsarbeit einnehmen $^{1}$, werden andere Formen der Auftragskommunikation - wie Werbung, Sponsoring oder Direktmarketing - von kirchlichen Einrichtungen nur zögerlich akzeptiert. Sei es, daß eine Kirchengemeinde Kugelschreiber und T-Shirts mit ihrem Logo bedrucken lassen will; sei es, daß eine Pfarrerserie im Privatfernsehen aus Kirchenmitteln gefördert werden soll; sei es, daß mehrere deutsche Bistümer eine PR- und Werbeagentur mit der offentlichkeitswirksamen Verbreitung religiöser Botschaften beauftragen - mit Blick auf die möglichen Vor- und Nachteile gerăt meist schon im Vorfeld nicht nur das jeweilige Projekt, sondern regelmäßig das Verhältnis Kirche und Werbung als solches in die Diskussion. ${ }^{2}$

Bestimmte Fragen tauchen dabei in der einen oder anderen Variante immer wieder auf:

- Darf sich die Kirche auch solcher Kommunikationsmittel bedienen, die bezahlt werden müssen und üblicherweise zu kommerziellen Zwecken eingesetzt werden?

- Läßt sich der umfassende, im Evangelium begründete Kommunikationsauftrag der Kirche an untergeordnete oder ausgelagerte Stellen (z.B. eine Werbeagentur) delegieren?

- Gerät die damit angestrebte Professionalisierung nicht in Konflikt

Dr. Michael Krzeminski M.A. ist Kommunikationswissenschaftler an der Universität Gesamthochschule Siegen und unterrichtet dort im Diplomstudiengang Medien-Planung, -Entwicklung und -Beratung.

1 Die erste Pressestelle der katholischen Kirche wurde bereits 1916 in New York eingerichtet. Vgl. hierzu und zur weiteren Entwicklungen diözesaner Öfentlichkeitsarbeit Manfred Becker-Huberti, "Kirchliche Presse- und Offentlichkeitsarbeit im Erzbistum Köln“, in: Praxis des Social Marketing, Michael Krzeminski/Clemens Neck ( $\mathrm{Hg}$.), Frankfurt/M.: Institut für Medienentwicklung und Kommunikation 1994, 99-124.

2 Ein Beispiel ist die Auseinandersetzung um eine gemeinsame Werbekampagne der Diözesen Limburg, Mainz, Speyer und Trier in Verbindung mit der Frankfurter Agentur ABC/Eurocom. Vgl. dazu Berichte im Rheinischen Merkur Nr. 44 v. 29.10.1993, S. 23 sowie das Interview mit P. Raban Tilman in Multimedia (Wien) v. 06.03.1994. 
mit Kommunikationszielen der Kirche wie Glaubwürdigkeit und Authentizität?

Leider bleibt die Debatte solcher Fragen häufig von Unkenntnis und Vorurteilen über Funktion und Verfahrensweisen der Auftragskommunikation überschattet. Insbesondere die Werbung wird gern für mißliebige kulturelle oder gesellschaftliche Entwicklungen verantwortlich gemacht, die sie allenfalls mitbeeinflussen, in den seltensten Fällen aber auslösen oder verhindern kann. ${ }^{3}$ Darüber hinaus werden die Begriffe Werbung, PR und Marketing selbst von Fachleuten unsystematisch oder gar synonym gebraucht, obwohl sich dahinter ein differenziertes, zunehmend auch besser erforschtes publizistisches Praxisfeld eröffnet.

Die nachstehenden Ausführungen sollen zu einer Versachlichung der Diskussion beitragen und den Blick dafür schärfen, daß die Formen der Auftragskommunikation es verdienen, neben denjenigen der künstlerischen oder journalistischen Medienproduktion unter den publizistischen Arbeitsfeldern der Kirche eine angemessene Berücksichtigung zu finden. Dies erscheint um so dringlicher, als die aktuelle Entwicklung des Mediensystems der industrialisierten Gesellschaften einen raschen Zuwachs im Angebot sowie in der Nachfrage bezahlter Kommunikationsdienstleistungen erwarten läßt. Die Kirche muß sich dieser Entwicklung stellen und darüber entscheiden können, für welche ihrer sozialkommunikativen Aufgaben sich Mittel der Auftragskommunikation eignen und für welche nicht. Die Formen der Auftragskommunikation (Werbung, PR, Marketingkommunikation) sind in dieser Hinsicht möglicherweise unterschiedlich zu bewerten und deshalb untereinander sowie von journalistischen Arbeitsformen klar abzugrenzen. Auf dieser Grundlage sollte es abschließend möglich sein, auch hinsichtlich der oben genannten Fragen sachgerechte und praxisnahe Antworten zu finden.

\section{Bedeutungszuwachs der Auftragskommunikation}

Ohne in die Fortschrittseuphorie zu verfallen, die gegenwärtig manche Medienfachtagung beherrscht, muß man selbst bei nüchternster Betrachtungsweise feststellen, daß sich die Medien der sozialen Kommunikation zur Zeit in einem Maße und mit einer Geschwindigkeit verändern, die den schon etwas abgegriffenen Topos vom "Sprung in das Informationszeitalter" gerechtfertigt erscheinen lassen. Vergleichbar ist diese Entwicklung am ehesten mit derjenigen nach Einführung und massenhafter Verbreitung des Automobils; das Schlagwort von den

3 Vgl. den Bericht von Susanne Kampmann über eine Akademietagung zum Thema "Werbung und Ethik" in: Communicatio Socialis 26 (1993) Heft 1, $59-63$. 
Datenautobahnen, auf denen sich neben der Industrie zunehmend auch private PC-Benutzer freizügig bewegen können, gewinnt seinen Reiz aus dieser Analogie. Es liegt auf der Hand, daß mit der weiteren Verbreitung der Datentechnik und dem Ausbau des Datenverkehrs vor allem auch deren kommerzielle Nutzung einem enormen Wachstumspotential entgegenblickt.

Es gibt indessen noch eine weitere, eher sozialpsychologische Begründung für die zunehmende Bedeutung gewerblicher Kommunikationsdienstleistungen in der Informationsgesellschaft. Verschiedene Kommunikationsforscher haben in den letzten Jahren darauf hingewiesen, daß der gegenwärtig zu beobachtende Wandel im Mediensystem der industrialisierten Gesellschaften unter anderem deshalb so schnell und drastisch erscheint, weil mehrere Entwicklungstrends ineinandergreifen und sich wechselseitig verstärken. Denis McQuail hebt in diesem Zusammenhang die vier Aspekte Internationalisierung, Informatisierung, das Entstehen einer postmodernen Kultur ohne verbindliche Werte und Individualisierung hervor. ${ }^{4}$ Michael Schmolke hat in dieser Zeitschrift auf die Omnipräsenz der elektronischen Medien, die Überkomplexität medialer Botschaften und die Wahlfreiheit des Publikums hingewiesen. ${ }^{5}$

Der Nürnberger Kommunikationswissenschaftler Winfried Schulz schließlich stellt für die vergangene Dekade insbesondere die folgenden Entwicklungstrends heraus:

- Technologische Integration, d.h. das Zusammenwachsen solcher Bereiche wie Personalcomputer und TV zu Multimedia-Systemen, oder die Entwicklung der CD zum gemeinsamen Ton-, Bild- und Datenträger;

- Internationalisierung, d.h. zunehmende Verflechtung nicht nur der Volkswirtschaften, sondern auch der Hardware- und SoftwareProduzenten mit erheblichen weltweiten Konzentrationsprozessen;

- Kommerzialisierung von Radio und Fernsehen, d.h. Deregulierung des vormals offentlichen oder staatlichen Rundfunks in Europa und in anderen Teilen der Welt, Zunahme der Hörfunk- und Fernsehwerbung, Programmgestaltung unter dem Gesichtspunkt von Marktfähigkeit und Nutzerakzeptanz;

- Angebotsexplosion, d.h. ernorme Vervielfältigung des Angebots an Radio- und Fernsehprogrammen, aber auch bei Publikumszeitschriften, bei CD und Video, bei Nachrichtenagenturen und Datenbanken.

4 Denis McQuail, Mass Communication Theory. An Introduction, London: Sage 31994, 26ff.

5 Michael Schmolke, "Kirchliche Kommunikation in der entwickelten Kommunikationsgesellschaft", in: Communicatio Socialis 26 (1993) 26-39. 
Als Ergebnis dieser Entwicklungen beobachtet Schulz eine voranschreitende Ausdifferenzierung des Publikums, d.h. durch die Vervielfältigung des Angebots und die Individualisierung der Wahlmöglickeiten werden die von den Massenmedien simultan erreichten Nutzergruppen immer kleiner. 6

Die hier beschriebenen Tendenzen lassen sich durchaus als Richtungsachsen in einem Kräfteparallelogramm verstehen, dessen Resultante der gegenwärtige Modernisierungsschub im Mediensektor ist. Darauf deuten auch eine Reihe von Wechselwirkungen zwischen den erwähnten Faktoren hin:

- Durch die Integration verschiedener Kommunikationstechniken verschwimmt die Grenze zwischen Individualkommunikation und Massenkommunikation zugunsten eines Kontinuums von Telekommunikationsformen mit unterschiedlicher Reichweite und graduell abgestufter Interaktivität (Beispiel: Telefon, Fax-Polling, Mailboxen, Online-Dienste).

- Die weltweiten Konzentrationsprozesse bei den Hardware- und Software-Herstellern begünstigen zwar einerseits die Tendenz zur internationalen Standardisierung von Medienprodukten und Dienstleistungen, bringen andererseits aber auch neue Formen der Angebotsdiversifizierung hervor (z.B. internationaler Rundfunk und Spartenfernsehen, "Weltmusik" und "Ethno-Rock").

- Die "Kommerzialisierung" und Deregulierung der europäischen Rundfunksysteme ist wesentlich mitverursacht durch eine allgemein auf Deregulierung ausgelegte Wirtschaftspolitik, die gegenwärtig auch andere gesellschaftliche Dienstleistungsbereiche betrifft, z.B. das Post- und Beförderungswesen, Sicherheit, kommunale Dienstleistungen, das Bildungssystem u.a.m.

- Die Vervielfältigung des Medienangebots schließlich induziert eine Steigerung der Nachfrage und schafft damit einen Anreiz für die weitere technische Entwicklung und technologische Integration (Beispiel DTP, CD-ROM).

Zusammenfassend läßt sich feststellen: Nicht nur das Informationsangebot der publizistischen Medien, sondern auch das Leistungsangebot von Wirtschaftsunternehmen, Behörden, Institutionen und sonstigen Organisationen erreicht die Menschen zu einem immer geringeren Teil in ihrer Eigenschaft als Mitglieder einer allgemeinen oder nach großen Interessengruppen gegliederten Öffentlichkeit. $\mathrm{Zu}$ einem immer größeren Teil werden sie hingegen als Angehörige kleinerer und wechselnder sozialer Segmente angesprochen, die sich für

6 Winfried Schulz, "Medienwirklichkeit und Medienwirkung. Aktuelle Entwicklungen der Massenkommunikation und ihre Folgen “, in: Aus Politik und Zeitgeschichte - Das Parlament, B 40 (1993), 16-26. 
bestimmte Themen, Produkte oder Dienstleistungen interessieren. ${ }^{7}$ Das wiederum bringt zahlreiche neue Dienstleistungsformen im Bereich der gesellschaftlichen Kommunikation hervor. Der Zürcher Kommunikationsforscher Ulrich Saxer hat in diesem Zusammenhang auf den Funktionszuwachs der Public Relations hingewiesen, die zunehmend auch solche Aufgaben integrieren, die traditionell dem Journalismus oder der Werbung vorbehalten waren. ${ }^{8}$ Die Ausdifferenzierung der Öffentlichkeit ist somit einerseits Ergebnis, andererseits aber auch Faktor der aktuellen Entwicklungen im Mediensystem.

Für die Kommunikationsplanung und -gestaltung solcher Einrichtungen, die nicht im Verborgenen blühen, sondern im öffentlichen Leben wirken wollen oder müssen, hat das mindestens die folgenden Konsequenzen:

- Immer mehr Institutionen und Einrichtungen, die früher als selbstverständlich oder selbsterklärend galten - angefangen bei Spitzenbehörden des Staates über Selbsthilfeeinrichtungen wie Krankenkassen bis hin zum ortsnahen Altenpflege-Service - sind mittlerweile erklärungsbedürftig geworden und müssen sich einer zunehmend ausdifferenzierten Offentlichkeit verständlich machen. Damit wächst die Konkurrenz um öffentliche Aufmerksamkeit und Beachtung in den Medien um ein Vielfaches - entsprechend schwieriger wird es, sich in dieser Konkurrenz zu behaupten.

- Wirtschaftswerbung und Unternehmens-PR haben professionelle Standards in Mediaplanung und Kommunikationsgestaltung gesetzt, an denen sich auch die Öffentlichkeitsarbeit von Verbänden und Nonprofit-Organisationen orientiert. Ohne das entsprechende Expertenwissen kann der öffentliche Auftritt einer Organisation oder Bewegung auf Dauer nicht erfolgreich sein.

- Selbst einfache Kommunikationsziele wie die Spendenwerbung für Kriegsopfer oder der Absatz eines Dritte-Welt-Kaffees lassen sich unter heutigen Bedingungen nur noch mit großem Aufwand und komplexen Mitteln bewältigen, z.B. im Medien- und Produktverbund oder durch die zeitliche und sachliche Kombination unterschiedlicher Kommunikationsmaßnahmen in einer Kampagne oder Strategie. ${ }^{9}$

7 In diesem Zusammenhang ist auch der häufig beklagte Mitgliederschwund bei den großen Werte- und Gesinnungsgemeinschaften (politische Parteien, Gewerkschaften, Kirchen) zu sehen. Offensichtlich verlieren dauerhafte weltanschauliche Bindungen an Attraktivität, während der Anteil z.B. von Wechselwählern oder zeitweiligen Sympathisanten wächst. In der Absatzwirtschaft wird eine analoge Tendenz weg von der Markentreue hin zum "multioptionalen" Konsumenten beobachtet.

8 Ulrich Saxer, "Public Relations als Innovation", in: Media Perspektiven (1991) Heft 5, 273-290.

9 Vgl. Christoph Zeller, "Alles aus einer Hand. Integrierte Kommunikation im Spendenmarketing ", in: Praxis des Social Marketing, Michael Krzeminski/ 
Es liegt auf der Hand, daß gesellschaftsbezogene Kommunikationsziele unter diesen Umständen nur noch mit größtem fachmännischen Eifer und professionellem Know-how zu erreichen sind. Ohne die verschiedenen Formen der Auftragskommunikation und ihre wirtschaftlichen Grundlagen wiederum ist die Entfaltung dahingehender Professionalität nicht vorstellbar. Es wäre deshalb durchaus eine Überlegung wert, den Typus der Auftragskommunikation auch im formalen Spektrum publizistischer Arbeitsfelder der Kirche stärker zu berücksichtigen und organisatorisch einzubinden - zumal die bisherige Aufteilung in Presse (UCIP), Film (OCIC) und Rundfunk (Unda) angesichts des zukünftigen Leitmediums Computer ohnehin zu Abgrenzungsschwierigkeiten führt und eine Reorganisation erforderlich erscheinen läßt. ${ }^{10}$ Dieses Problem soll uns hier jedoch nicht vordringlich beschäftigen. Naheliegender erscheint es, einige konkrete Beurteilungskriterien zu erarbeiten, mit deren Hilfe an Bischofssitzen und in Pfarreien, in kirchlichen Werken und in Laienverbänden über Sinn und Unsinn von Werbung, PR und Marketing-Kommunikation für Kommunikationsziele der Kirche reflektiert und entschieden werden kann.

\section{Arbeitsfelder der Auftragskommunikation}

Von der früheren Deutschen Bundesbahn wird kolportiert, daß keines ihrer bekannten Anzeigenmotive in Bahnhöfen oder Unterführungen plakatiert gewesen sein soll, weil sich die Abteilungen "Außenwerbung" und "Verkaufsförderung" nicht über die Zuständigkeit einigen konnten. ${ }^{11}$ Was immer die Absicht solcher Spitzfindigkeit gewesen sein mag - klar ist, daß die Definition von Arbeitsfeldern im Bereich der Auftragskommunikation auch unter Fachleuten nicht unstrittig ist. Um so weniger sollte es erstaunen, daß Begriffe wie Reklame, Werbung, Verkaufsförderung und Marketing im Alltagssprachgebrauch kaum deutlich abgegrenzt oder gar völlig synonym verstanden werden. Wahrscheinlich bereitet das in vielen Fällen auch gar keine Probleme. Für den hier verfolgten Argumentationszusammenhang erscheint es

Clemens Neck (Hg.), Frankfurt/M.: Institut für Medienentwicklung und Kommunikation 1994, 181-192.

10 Immerhin hat die explosionsartige Verbreitung des Videorecorders in allen Teilen der Welt vor gut einem Jahrzehnt zwischen den für Film und Fernsehen zuständigen kirchlichen Weltorganisationen (OCIC und Unda) zunächst für ein gewisses Kompetenzgerangel - und in der Folge für eine intensivere Zusammenarbeit gesorgt.

11 Unter Verkaufsförderung werden vor allem kurzfristige zusătzliche Anreize zur Absatzförderung verstanden (Sonderangebote, Rabatte, Inzahlungnahmen, Zugaben) sowie absatzsteigernde Maßnahmen am eigentlichen Verkaufsort (Hinweisschilder im Regal, Sondertische, Displays in Kassennähe, Verteilung von Warenproben und Produktdemonstrationen im Supermarkt oder in der Fußgängerzone). Vgl. Heribert Meffert, Marketing. Grundlagen der Absatzpolitik, Wiesbaden: Gabler 1977 ff. 
allerdings von Bedeutung, sich zumindest einiger grundlegender Definitionsmerkmale zu vergewissern.

\subsection{Werbung}

"Der Einfluß der Werbung auf den Menschen in der heutigen Zeit wächst von Tag zu Tag, und niemand kann sich ihrer Wirkung entziehen. Sie bringt der Gesellschaft vielfachen Nutzen. Der Käufer wird durch sie informiert über Sachgüter, die er benötigt, und über Dienstleistungen, die zur Verfügung stehen. So wird durch Werbung eine breite Verteilung der Produktionsgüter ermöglicht. Das wiederum verhilft der Industrie zu weiteren Fortschritten, die sich erneut zum Besten der ganzen Gesellschaft auswirken. Darum ist gegen Werbung nichts einzuwenden, wenn sie auf ihre Weise der Wahrheit verpflichtet bleibt und die Wahlfreiheit des Käufers wahrt, auch in den Fällen, wo man, um seine Kauflust anzuregen, an seine elementaren Bedürfnisse appelliert und eine Ware als unbedingt nötig anpreist." 12

Diese Beschreibung stammt nicht etwa aus einem wirtschaftspädagogischen Lehrbuch, sondern aus der Pastoralinstruktion "Communio et Progressio" (1971), dem inzwischen durch ein Nachfolgedokument ("Aetatis novae", 1992) ergänzten, in vielem jedoch immer noch maßgeblichen offiziellen Dokument der katholischen Kirche zu den ,Instrumenten der sozialen Kommunikation'. Ich will nicht verhehlen, daB im Anschluß an diesen Passus noch viel Kritisches und Warnendes zum Umgang mit der Werbung ausgesprochen wird. Die grundsätzliche Anerkennung der Existenzberechtigung der Werbung sowie ihrer Notwendigkeit für eine funktionierende Marktwirtschaft erscheint mir indessen besonders beachtenswert, zumal sie in der fast fünfundzwanzigjährigen Rezeptionsgeschichte dieses Dokuments nur wenig Aufmerksamkeit gefunden hat.

Werbung ist dieser und anderen Beschreibungen zufolge eine spezielle Form der Informationsübermittlung mit überwiegend appellativem Charakter, die insofern eine gewisse Zwitterstellung einnimmt, als sie elementarer Bestandteil sowohl des Wirtschafts- als auch des Kommunikationssystems moderner Gesellschaften ist. Ohne Werbung ließen sich weder die Fortschritte im Automobilbau noch eine unabhängige Presse finanzieren. Werbung kann darüber hinaus als Indikator der kulturellen Entwicklung sozialer Gemeinschaften betrachtet werden, der Aufschluß über Strömungen des Zeitgeistes oder längerfristige Prozesse des Einstellungs- und Wertewandels gibt. ${ }^{13}$ Hier wäre beispielsweise die

12 Pastoralinstruktion "Communio et Progressio über die Instrumente der sozialen Kommunikation, veröffentlicht im Auftrag des II. Vatikanischen Ökumenischen Konzils 1971, Artikel 59.

13 Vgl. zu diesem Analyseansatz Siegfried J. Schmidt, "Werbewirtschaft als soziales System", in: Arbeitshefte Bildschirmmedien 27, Siegen: DFG- 
Frage anzusiedeln, welchen Einfluß die Werbung auf die Ausprägung hedonistischer Konsumgewohnheiten nimmt, aber auch, welchen Beitrag sie möglicherweise zur Überwindung von Prestigedenken und zur Auflösung verfestigter Statussymbole leisten kann.

Tatsächlich werden am Beispiel der Werbung häufig Probleme verhandelt, die das Kommunikationssystem der Gesellschaft als solches betreffen oder einzelne Medienkanäle, in denen Werbung eine Rolle spielt, z.B. die Gattung der Publikumszeitschriften, der Kinofilm oder das Fernsehen. Denn letztendlich sind es nur wenige Merkmale, mit denen sich Werbung von anderen Erzeugnissen der Publizistik unterscheidet: Der ausschlaggebende Grund für ihr Erscheinen ist, daß jemand dafür bezahlt und sich mit einer bestimmten Botschaft zu erkennen gibt. Andere Eigenschaften der Werbung - wie Interessegebundenheit, Persuasivität, Emotionalität, gegebenenfalls auch Suggestivität - lassen sich auch bei anderen publizistischen Produkten beobachten; im Prinzip auch bei solchen, die man gewöhnlich dem Journalismus zuordnet.

Es erscheint demnach triftig, sich bei einer Definition auf die wenigen unverwechselbaren Merkmale zu stützen, bei deren gemeinsamen Auftreten man etwas als Werbung erkennt: Werbung ist die Vermittlung einer Botschaft auf bezahltem öffentlichen Raum, die von einer identifizierbaren Quelle kommt. Diese Basisdefinition berücksichtigt die vielfältigen Erscheinungsformen der Werbung - vom TV-Spot über die Stadionwerbung bis zum Kultursponsoring, dessen spezielle Botschaft darin besteht, daß sich ein Auftraggeber in seiner Selbstdarstellung zugunsten der geförderten Sache zurückhaltender als bei der "normalen“ Werbung verhält. Darüber hinaus läßt sie der Tatsache Raum, daß prinzipiell alles zum Gegenstand der Werbung werden kann: Vom Vollwaschmittel bis zur staatlichen Aufklärung über die Rentenreform. Die Definition stellt außerdem die besondere Verantwortlichkeit des Auftraggebers für seinen öffentlichen Auftritt heraus: Tatsächlich kann ich in jedem Fall von Werbung davon ausgehen zu wissen, wer etwas von mir will. Wenn Verwechslungsgefahr mit redaktionellen Beiträgen besteht, muß Werbung sogar besonders gekennzeichnet sein. Im Interesse der Meinungsfreiheit ist die Verbreitung journalistischer Botschaften längt nicht im gleichen Maße reglementiert. ${ }^{14}$

Das heißt natürlich nicht, daß Werbung nicht übertreiben,

Sonderforschungsbereich 240/Universität GH Siegen 1991; sowie Siegfried J. Shmidt/Brigitte Spieß (Hg.), Die Geburt der schönen Bilder. Fernsehwerbung aus der Sicht der Kreativen, Opladen: Westdeutscher Verlag 1994.

14 Diesem Umstand ist es beispielsweise zu verdanken, daß die berüchtigte Werbekampagne des italienischen Textilfabrikanten Benetton nunmehr auch gerichtlich sanktioniert worden ist, unabhängig davon, daß sie sich wirtschaftlich als kontraproduktiv erwiesen hatte. 
beschönigen oder dem Adressaten schmeicheln dürfte. Im Anreiz zum Verkaufen liegt ihr wirtschaftlicher und gesellschaftlich akzeptierter Sinn. Im Mittelpunkt der Werbebotschaft steht demnach stets ein ausgesprochenes oder unausgesprochenes Nutzenversprechen. ${ }^{15}$ Genau deshalb darf Werbung allerdings auch nicht wirklich lügen - nichts schadet dem Anbieter einer Ware oder Dienstleistung auf Dauer mehr als eine getäuschte oder enttäuschte Kundschaft.

Dabei ist zu berücksichtigen, daß die Herstellung von Markttransparenz, d.h. die Information über Anbieter, Angebote und Preise von Waren und Dienstleistungen auch in der Überflußgesellschaft immer noch den Hauptzweck der Werbung ausmacht. Der größte Anteil der Werbeeinnahmen wird in den regionalen Tageszeitungen erzielt, u.a. durch die Inserate der örtlichen Handwerksbetriebe bzw. die Beilagen der Möbelhäuser und Supermärkte mit den Sonderangeboten der Woche. Die vielzitierten und viel kritisierten "geheimen Verführer", die uns per Kino- und Fernsehwerbung ein Leben voller Abenteuer, Erfolg und Partnerglück versprechen, wenn wir eine bestimmte Zigaretten-, Putzmittel- oder Duftwasser-Marke verwenden, haben wirtschaftlich nicht die gleiche Bedeutung, obgleich die Auftragssummen im einzelnen natürlich größer sind.

\section{2 Öffentlichkeitsarbeit und Public Relations}

Das Besondere der Öffentlichkeitsarbeit gegenüber der Werbung kommt vielleicht am anschaulichsten in dem folgenden Aphorismus zum Ausdruck: "Wenn ein junger Mann einem schönen Mädchen sagt, was für ein toller Kerl er sei, ist das Reklame; wenn er ihm sagt, wie reizend er es findet, ist das Werbung. Wenn er jedoch seine Freunde dazu bringt, dem Mädchen zu sagen, was für ein toller Kerl er sei, ist das Public Relations". Öffentlichkeitsarbeit - der deutsche Begriff kann als bedeutungsgleich mit dem international gebräuchlichen Public Relations gelten - meint also mehr als die Befolgung der weitverbreiteten Maxime „Tue Gutes und rede darüber!". Öffentlichkeitsarbeit kann vielmehr als der Oberbegriff für ein ganzes Bündel von Maßnahmen der Kommunikation von Organisationen angesehen werden, deren gemeinsames Merkmal darin besteht, daß sie sich an ein disperses, im persönlichen Kontakt nicht oder nur schwer erreichbares Publikum wenden. Dazu gehören normalerweise Bezugsgruppen in der äußeren Umwelt einer Organisation (Kunden, Lieferanten, Anwohner, Multiplikatoren, Medienvertreter, etc.), es können aber auch solche der internen Umwelt sein (Mitglieder, Mitarbeiter, Anteilseigner, etc.).

Der Beginn moderner Öffentlichkeitsarbeit wird üblicherweise an der Bestellung hauptberuflicher Pressesprecher durch amerikanische Bergbau- und Eisenbahnunternehmen in der Zeit um die Jahrhundert-

15 Vgl. David Ogilvy, Ogilvy über Werbung, Düsseldorf: Econ 1984. 
wende festgemacht. Kein anderer als John D. Rockefeller sen. engagierte in dieser Funktion schon 1905 den später berühmt gewordenen Ivy Lee, hauptsächlich infolge einer immer heftiger werdenden kritischen Presseberichterstattung über die Mineralöl- und Anthrazitkohleindustrie. Ivy Lee gab als erster eine Art Prinzipienkodex heraus ("Unser Plan ist es, kurz und offen, die Presse und die Bevölkerung schnell und genau über die Tatsachen zu unterrichten, die für sie von Wert und Interesse sind"); 1914 wurde er von John D. Rockefeller jun. als offizieller PR-Assistent der Standard Oil Company eingesetzt und gründete daraufhin die PR-Agentur Lee, Harris \& Lee. ${ }^{16}$

Ein weiterer Ziehvater der professionellen PR, Edward L. Bernays, begann seine Karriere 1914 und wurde von US-Präsident Woodrow Wilson in das "Comittee on Public Information" eingesetzt, um für die Kriegsziele der USA im fernen Europa und die Zeichnung von Friedensanleihen zu werben. Mit seinem verständigungsorientierten Ansatz der Informationspolitik setzte sich Präsident Wilson erfolgreich gegen Forderungen der amerikanischen Heeres- und Marineleitung nach Zensurmaßnahmen durch. Edward L. Bernays brachte seine Berufserfahrung später vor allem in Wissenschaft und Ausbildung ein. In Deutschland wurden die Public Relations insbesondere durch Carl Hundhausen und Albert Oeckl bekanntgemacht; letzterer führte als Leiter der entsprechenden Abteilung des Deutschen Industrie- und Handelstages 1951 den Begriff "Öffentlichkeitsarbeit" ein.

$\mathrm{Zu}$ ihrer heutigen überragenden Bedeutung - ohne systematische Öffentlichkeitsarbeit läßt sich in der Informationsgesellschaft kaum noch wirtschaftlicher, politischer oder sozialer Einfluß geltend machen fanden die Public Relations also in einem jahrzehntelangen Prozeß. Historisch-typologisch lassen sich die folgenden Entwicklungsstufen unterscheiden:

- Öffentlichkeitsarbeit als reaktives „Reinwaschen“ und „Übertünchen “ von Kritik (in der Gründerzeit).

- Öffentlichkeitsarbeit als punktuelle Erzeugung öffentlicher Aufmerksamkeit für Absatzförderung und Imageverbesserung (in der entstehenden Konsumgesellschaft).

- Öffentlichkeitsarbeit als der Versuch der Interessenharmonisierung zwischen Wirtschaft und Bevölkerung (im Nachkriegsdeutschland).

- Öffentlichkeitsarbeit als kontinuierliche und systematische Beziehungspflege mit den unterschiedlichen Zielgruppen eines Unternehmens (in der Gegenwart). ${ }^{17}$

16 Vgl. Albert Oeckl, „Anfänge und Entwicklung der Öfentlichkeitsarbeit“, in: Public Relations/ Öffentlichkeitsarbeit, Heinz-D. Fischer/Ulrike Wahl ( $\mathrm{Hg}$.), Frankfurt/M.: Peter Lang 1993, 15-31.

17 Vgl. Günter Barthenheier, „Auf der Suche nach Identität - Zur historischen Entwicklung der Öfentlichkeitsarbeit/Public Relations“, in: Öffentlichkeits- 
Aufgrund dieses Funktionszuwachses bemühen sich zahlreiche aktuelle Arbeiten zum Thema um die theoretische Herleitung und praktische Begründung von Public Relations als eigenständiger Forschungs- und Unterrichtsdisziplin. ${ }^{18}$ Ulrich Saxer hat bereits 1991 vorgeschlagen, Public Relations als ein gesellschaftliches Innovationssystem zu betrachten, das auf dem besten Wege sei, sich von einem Hilfsinstrument der Unternehmenskommunikation über die Ausdifferenzierung als publizistisches Teilsystem industrieller Gesellschaften zu einer Art Leitsystem der Gesellschaftskommunikation zu entwickeln, das zunehmend auch solche Aufgaben integriert, die traditionell der Werbung oder dem Journalismus vorbehalten waren.19 Die mittlerweile eingetretene Beschleunigung der Entwicklung gibt dieser Prognose insofern recht, als die hier genannten publizistischen Arbeitsfelder zunehmend stärker diffundieren und funktionale Äquivalenzen herausbilden.

Einig sind sich Vertreter aus Theorie und Praxis darüber, daß erfolgreiche Öffentlichkeitsarbeit proaktiv, kontinuierlich und systematisch betrieben werden muß, während reaktive, defensive und sporadische Aktionen eher schaden. Darüber hinaus wird immer wieder auf den dialogischen Charakter der PR hingewiesen. Öffentlichkeitsarbeit ist ein zweiseitiger Kommunikationsprozeß, der einerseits der Vermittlung bestimmter Images dient, andererseits aber auch die Anpassung eines Unternehmens oder einer Organisation an die Vorstellungen und Wünsche der betreffenden Zielgruppe ermöglichen soll. „Public relations", so die klassische Definition von Edward L. Bernays, "is the attempt, by information, persuasion, and adjustment, to engineer public support for an activity, cause, movement, or institution." 20 Erfolgreiche Öffentlichkeitsarbeit beginnt deshalb mit und beinhaltet immer wieder auch ein sorgfältiges Hinhören und Zuhören; nicht zufällig ist die systematische Beobachtung und Analyse der öffentlichen bzw. der veröffentlichten Meinung ein zentraler Aufgabenbereich der entsprechenden Fachabteilungen. ${ }^{21}$

arbeit. Dialog zwischen Institution und Gesellschaft, Günther Haedrich/ Günter Barthenheier/Horst Kleinert (Hg.), Berlin: de Gruyter 1982.

$18 \mathrm{Vgl}$. insbesondere Horst Avenarius/Wolfgang Armbrecht (Hg.), Ist Public Relations eine Wissenschaft? Eine Einführung, Opladen: Westdeutscher Verlag 1992, sowie Franz Ronneberger/Manfred Rühl, Theorie der Public Relations. Ein Entwurf, Opladen: Westdeutscher Verlag 1992.

19 Vgl. Ulrich Saxer, "Public Relations als Innovation“, in: Media Perspektiven (1991) Heft 5, 273-290, besonders S. 285.

20 Edward L. Bernays, The Theory and Practice of Public Relations - A Resume, in: The Egineering of Consent, Edward L. Bernays (Hg.), Norman: Oklahoma UP, 21956, S. 3.

21 Dazu gehört im weitesten Sinne auch die Kommunikationsforschung, wie sie in der Pastoralinstruktion "Aetatis novae" zwanzig Jahre nach "Communio et Progressio" in einem Anhang unter dem Titel "Elemente eines Pastoralplans für soziale Kommunikation“ dargestellt wird (1992, Artikel 32). In 
Allerdings wird die Dialogorientierung in der Öffentlichkeitsarbeit gelegentlich auch überbetont. Der gleichberechtigte (symmetrische) Dialog mit den Zielgruppen ist für die Öffentlichkeitsarbeit nur dann konstitutiv, wenn die Dialogorientierung selbst $\mathrm{zu}$ den obersten Organisationszielen gehört, beispielsweise im Fall einer Beratungsstelle oder eines Geselligkeitsvereins. Wirtschaftsunternehmen und solche Organisationen, die primär andere Zwecke verfolgen (Gewinnerwirtschaftung, Bestandserhaltung, sonstige materielle oder ideelle Ziele), sind durchaus legitimiert, den Dialog mit den Zielgruppen als Instrument für die eigene Informations- und Überzeugungsarbeit einzusetzen und auf das Zweckmäßige zu konzentrieren. ${ }^{22}$ Skepsis ist jedenfalls angebracht, wenn Dialogbereitschaft anderen Zielen der Öffentlichkeitsarbeit nur übergestülpt wird, obwohl offensichtlich ist, daß sie in erster Linie Mittel zum Zweck sein soll.

\subsection{Social Marketing}

Das breiteste und vielfältigste unter den hier vorgestellten Arbeitsfeldern ist die Kommunikation im Social Marketing. Der Begriff wird auf einen Aufsatz des amerikanischen Kommunikationsforschers Gert D. Wiebe (1952) zurückgeführt, der die Frage behandelt, ob und unter welchen Bedingungen bestimmte Instrumente der Absatzförderung erfolgreich für die Popularisierung sozialer Wertvorstellungen wie Bürgersinn und nachbarschaftliches Engagement eingesetzt werden können. Dabei stellt er die in der Folge oft zitierte Frage: "Why can't you sell brotherhood and rational thinking like you sell soap?"23 Seitdem hält sich hartnäckig das Gerücht, im Social Marketing gehe es darum, soziale Wertvorstellungen mit den Mitteln der Konsumgüterwerbung an den Mann bzw. die Frau zu bringen. Eine erste theoretische Abhandlung zum Thema wurde 1972 von dem amerikanischen Marketing-Forscher Philip Kotler vorgelegt. ${ }^{24}$ In einer Vorstudie von P. Kotler und G. Zaltman finden wir die folgende Definition des von ihnen so bezeichneten "generischen" Marketing-Konzepts: "Social Marketing ist die Planung, der Einsatz und die Kontrolle von Programmen zur Beeinflussung der Akzeptanz von sozialen Vorstellungen, in die

diesem Dokument wird übrigens insbesondere auch die Unterhaltung von Büros der Kirche für Öfentlichkeitsarbeit gefordert (Artikel 31).

22 Ein Beispiel dafür ist die notwendige Professionalisierung des Umgangs mit Risiko. So versucht die Deutsche Lufthansa durch umfangreiche Maßnahmen in ihrer Presseabteilung sicherzustellen, daß sie im Fall eines Unfalls einer ihrer Maschinen irgendwo auf der Welt gegenüber den Medien als Erstinformant auftritt und sofort genügend Personal bereitstellen kann, um telefonische Anfragen von besorgten Angehörigen zu beantworten.

23 Gert D. Wiebe, "Merchandising Commodities and Citizenship on Television ", in: Public Opinion Quarterly, 15 (1952), 679-691, hier S. 679.

24 Vgl. Philip Kotler, "A Generic Concept of Marketing", in: Joumal of Marketing, 36 (1972), 46-54. 
Überlegungen zur Gestaltung des Produkts, des Preises, der Kommunikation, des Vertriebs und der Marketing-Forschung eingehen. "25

Diese Definition macht unter anderem zur Voraussetzung, daß soziale Vorstellungen zielgerichtet veränderbar sind und ihre Akzeptanz in der Gesellschaft sich beeinflussen läßt. Dieser Einfluß kann systematisch und kontrolliert ausgeübt werden. Auf dem „Marktplatz der Ideen" unterliegt der Austausch sozialer Vorstellungen ähnlichen Regeln wie der Austausch von Wirtschaftsgütern, d.h. er erfolgt nicht aufgrund von Zwang, Bettelei oder Räuberei, sondern als Folge eines freiwilligen Marktzutritts. Auch bei der Verbreitung sozialer Vorstellungen kommen also Merkmale wie deren Qualität, der "Preis", den man dafür zahlen muß, ihre Zugänglichkeit oder Verfügbarkeit sowie schließlich ihre Bekanntmachung durch Kommunikation ins Spiel. Der "Anbieter" einer sozialen Idee kann mithilfe dieser Kategorien die "Absatzchancen" seines Produkts bzw. seiner Dienstleistung ermitteln und durch gezielte Maßnahmen verbessern.

Kotler überträgt hier die Terminologie der klassischen MarketingLehre, die von den berühmten $4 \mathrm{P}$ des Marketing spricht (Product, Price, Place, Promotion), auf das Gebiet des Social Marketing. Dementsprechend werden auch in der deutschsprachigen Marketing-Forschung Produkt- bzw. Leistungspolitik, Preispolitik, Distributionspolitik und Kommunikationspolitik als zentrale Aufgabenfelder (oder Instrumentalbereiche) marktorientierter Unternehmensführung definiert. ${ }^{26}$ Durch die geeignete Kombination von Instrumenten, die in diesen Aktionsbereichen zur Verfügung stehen, sollen Kundenbedürfnisse besser erkannt und zielgruppengenau befriedigt werden. Voraussetzung dafür ist die kontinuierliche Markterfassung und Marktbearbeitung durch Marktsegmentierung und zielgruppenadäquate Positionierung. ${ }^{27}$

Im Social Marketing geht es darum, diese Prinzipien auch auf den freiwilligen Austausch immaterieller Güter wie soziale Leitbilder, Werte, politische Ideen oder religiöse Inhalte anzuwenden. In einer offenen Gesellschaft müssen solche Vorstellungen unter "Marktbedingungen", d.h. in Konkurrenz zu anderen und gegen mögliche Widerstände, zum Austausch gebracht werden. Austausch heißt in diesem Fall, daß von den "Abnehmern" solcher Leistungen auch Gegenleistungen erwartet werden, die selbst wiederum materieller oder immaterieller Natur sein können (Beiträge und Spenden, aber auch freiwilliger Arbeitseinsatz, Mitwirkung an der Selbstverwaltung, Mitglieder- und Sympathisantenwerbung u.ä.m.). Wie im Absatzmarketing werden die Qualität einer

25 Philip Kotler und Gerald Zaltman, "Social Marketing: An Approach to Planned Social Change", in: Joumal of Marketing, 35 (1971), 3-12, hier S. 5.

26 Vgl. Heribert Meffert, Marketing. Grundlagen der Absatzpolitik, Wiesbaden: Gabler 1977 u.ö.

27 Vgl. Hermann Freter, Marktsegmentierung, Stuttgart: Kohlhammer 1983. 
Idee, der Preis, den man dafür zahlen muß, die Zugänglichkeit und die Information über entsprechende Angebote durch Kommunikation als zentrale Instrumentalbereiche der Nachfrageforschung und Angebotsgestaltung angesehen.

Wichtig hervorzuheben ist, daß Kotler die gesellschaftlichen Austauschprozesse im Social Marketing als wechselseitige Interaktionen und keinesfalls nur als einseitige Persuasionsaufgabe sieht. Wie im Wirtschaftsleben steht der Anbieter einer sozialen Idee vor der Aufgabe, die Bedürfnisse seiner Abnehmer zu ermitteln, um sein Verhalten an diese Bedürfnisse anpassen zu können. „Eine verkaufsorientierte Haltung sieht ihre Aufgabe darin, Käufer für ein bestimmtes Produkt zu finden und sie zu überzeugen, dieses Produkt zu kaufen. [...] Das Marketing-Konzept dagegen fordert die höchste Einsatzbereitschaft gerade dafür, die Wünsche der Zielgruppe zu ermitteln, um dann die Produkte oder Dienstleistungen zu erzeugen, welche diese Wünsche befriedigen. In der Praxis sind natürlich zu jeder Zeit bestimmte Produkte schon da, während andere erst entwickelt werden, d.h. die meisten Marketing-Strategien sind eine Mischung aus Verkaufen und Marketing. . ${ }^{28}$

Es ist das Spannungsverhältnis von tatsächlichem und vorstellbarem Angebot, von tatsächlicher und vorstellbarer Nachfrage, aus der sich die Dynamik erklärt, die Märkte von anderen Verteilsystemen unterscheiden. Was spricht dagegen, für den Bereich der sozialen Vorstellungen anzunehmen, daß genau diese Dynamik und das damit verbundene Innovationspotential pluralistische Gesellschaften gegenüber totalitären Systemen auszeichnet? Social Marketing ist aus den vorgestellten Gründen tatsächlich nicht nur ein geeignetes Instrument der Unternehmensführung von Nonprofit-Organisationen, sondern in Ergänzung zum traditionellen Absatzmarketing auch ein sinnvolles Managementinstrument für Wirtschaftsunternehmen. AuBerdem sind die meisten Nonprofit-Unternehmen neben dem Social Marketing auch mit konkreten Absatzfragen befaßt. Dieses interessante Verhältnis wird an anderer Stelle ausführlicher diskutiert ${ }^{29}$ - hier ist vor allem festzuhalten, daß mit dem Social Marketing ein hervorragendes Planungsinstrument für das Management von Unternehmen und Organisationen zur Verfügung steht, die ihre Kommunikationsplanung als integrierten Bestandteil der Unternehmensplanung verstehen, und die ihre Kommunikationspolitik in Abstimmung mit Maßnahmen in den anderen Marketingbereichen (Produkt, Preis, Distribution) gestalten

28 Philip Kotler/Gerald Zaltman, a.a.O., S. 5; meine Übersetzung.

29 Vgl. Michael Krzeminski/Clemens Neck, "Social Marketing. Ein Konzept für die Kommunikation von Wirtschaftsunternehmen und Nonprofit-Organisationen ", in: Praxis des Social Marketing, Michael Krzeminski/Clemens Neck (Hg.), Frankfurt/M.: Institut für Medienentwicklung und Kommunikation 1994, 11-35. 
wollen. Nicht zufällig wirkt der öffentliche Auftritt vieler Unternehmen dann besonders stimmig und wahrhaftig, wenn ihre Kommunikationsmaßnahmen entweder auf einer natürlichen Corporate Identity bzw. auf einem systematisch erarbeiteten Unternehmensleitbild beruhen, das mithilfe des Marketingkonzepts entwickelt wurde.

\section{Auftragskommunikation für die Kirche}

Für die Diskussion der Frage, ob und in welcher Weise sich Formen der Auftragskommunikation für sozialkommunikative Aufgaben der Kirche eignen, erscheinen nach dem bisher Gesagten die folgenden Gesichtspunkte von besonderem Belang:

(1) Werbung ist die Vermittlung einer Botschaft auf bezahltem öffentlichen Raum, die von einer identifizierbaren Quelle kommt. Ihr Inhalt kann wahr oder falsch, die Quelle glaubwürdig oder unglaubwürdig sein; Werbezeit oder Werbefläche stehen grundsätzlich jedem $\mathrm{zu}$, der geschäftsfähig ist und dessen Botschaft nicht gegen Gesetze verstößt. Werbung ist an kein bestimmtes Medium gebunden, sondern stellt eine für Marktwirtschaften typische Kommunikationsinfrastruktur dar. Kritik an den Erscheinungsformen und Produkten der Werbung ist daraufhin zu prüfen, ob sie tatsächlich die Werbung oder Eigenschaften betrifft, die dem jeweiligen Trägermedium allgemein zuzuordnen sind.

(2) Öffentlichkeitsarbeit ist ein Oberbegriff für die von Unternehmen und Organisationen kontinuierlich und systematisch betriebene Kommunikation mit unterschiedlichen Zielgruppen in ihrer externen und internen Umwelt. Ohne Öffentlichkeitsarbeit ist in der Informationsgesellschaft kein politischer, wirtschaftlicher oder sozialer Einfluß möglich und der Bestand von Unternehmen und Organisationen ernsthaften Risiken ausgesetzt. Die Ausdifferenzierung der Funktion Öffentlichkeitsarbeit durch spezialisierte und professionelle Stabsabteilungen in allen nur denkbaren Organisationen ist Folge und zugleich verstärkender Faktor dieser Entwicklung. Mit dem Journalismus und dem Mediensystem insgesamt ist das System der Public Relations mittlerweile symbiotisch verzahnt.

(3) Social Marketing ist eine Perspektive der Unternehmensführung, die in Analogie zum Absatzmarketing ein Instrumentarium zur Erfassung und Bearbeitung von Angebots- und Nachfragesituationen hinsichtlich sozialer Werte und Einstellungen bereithält, das in die Dimensionen Qualität, Preis, Zugänglichkeit und Kommunikation gegliedert ist. Es geht von freien Austauschbeziehungen zwischen den Anbietern und Abnehmern sozialer Vorstellungen aus, anerkennt die Möglichkeit eines dahingehenden Wettbewerbs und fördert die Herausbildung einer Corporate Identity bzw. eines Organisationsleitbildes, durch welches die Maßnahmen zur Quali- 
tätsentwicklung, Preisgestaltung, Distribution und Kommunikation zum größtmöglichen Nutzen sowohl der Organisation als auch ihrer Klienten gewichtet und aufeinander abgestimmt werden.

Dergestalt auf das Wesentliche reduziert, ist festzuhalten, daß Werbung, Öffentlichkeitsarbeit und Social Marketing keine fakultativen oder sich wechselseitig ausschließenden Arbeitsfelder der Auftragskommunikation darstellen, sondern in einem Inklusionsverhältnis stehen. Während die Begriffe des Social Marketings zur Entwicklung von Kommunikationskonzepten herangezogen werden können, ist die Öffentlichkeitsarbeit für deren Umsetzung und die Werbung für den Transport der Botschaften an die Öffentlichkeit bzw. an bestimmte Zielgruppen zuständig. Tatsächlich müssen Unternehmen und Organisationen, die in der Informationsgesellschaft bestimmte Zwecke erreichen wollen, ihre Position im politischen, gesellschaftlichen und kulturellen Raum durch soziales Marketing definieren, sie sollten dazu Öffentlichkeitsarbeit betreiben und können hierfür Mittel der Werbung einsetzen.

Außerdem ist festzuhalten, $\mathrm{da} a$ den Arbeitsfeldern der Auftragskommunikation das gemeinsame Merkmal der Ziel- und Zweckgerichtetheit zu eigen ist. Auch wenn menschliche Kommunikation in jedem Fall sowohl Mittel ("means") als auch ein Ziel in sich selbst ("end") repräsentiert, werden in der Auftragskommunikation diesbezüglich klare Prioritäten gesetzt: Ausgangspunkt und Zielvorstellung aller Überlegungen und Aktivitäten der Auftragskommunikation sind die Probleme oder angestrebten Problemlösungen des Auftraggebers und nicht normative Vorstellungen über das Wesen oder die Funktionen der sozialen Kommunikation. Das unterscheidet die Arbeitsfelder der Auftragskommunikation von denen des Journalismus, die sich aus einer dahingehenden Normativität begründen lassen, sowie von denjenigen der künstlerischen Medienproduktion, welche die Zweckfreiheit der Kunst für sich in Anspruch nehmen.

Die strikte Zielunterwerfung bedeutet allerdings nicht, daß die Auftragskommunikation keine normativen Regeln befolgt und sich ethischen Begründungen oder moralischen Urteilen entzieht. Das Gegenteil ist der Fall: Eine Crux der öffentlichen Diskussion über den Einsatz von Werbung, PR und Marketing liegt darin, daß sie den elaborierten Kodex berufsethischer Standards auf diesem Gebiet, formuliert beispielsweise durch den deutschen Werberat oder die Deutsche Gesellschaft für Public Relations (DPRG), kaum ernsthaft zur Kenntnis nimmt. Allerdings - dahingehende Unterschiede sollten nicht verwischt werden - sind diese Standards andere als solche für den Journalismus, so wie für ein Fußballspiel andere Regeln gelten als für ein Volkstanzturnier. Niemand aus der Branche der Auftragskommunikation hat etwas dagegen, wenn seine berufsethischen Standards öffentlich - oder besser: mit ihm gemeinsam - diskutiert werden; allerdings sollte bei der normativen Begründung solcher Standards die Bereichszugehörigkeit respektiert und die auf Wettbewerbsvorteile 
bedachte Kommunikation an ihren eigenen (sozial sanktionierten) Spielregeln gemessen werden und nicht an solchen, die sich für Kommunikationsformen mit gänzlich anderer Zielsetzung etabliert haben. ${ }^{30}$

Die Frage, ob Formen der Auftragskommunikation für sozialkommunikative Aufgaben der Kirche taugen, stellt sich angesichts ihrer überragenden Bedeutung für die moderne Informationsgesellschaft tatsächlich nicht als eine Frage des "Entweder-Oder" sondern als eine Frage des "Wie". Dabei kann nach den Erscheinungsformen differenziert werden, in denen Kirche den Gläubigen sowie Außenstehenden hauptsächlich gegenübertritt: erstens als Glaubensinstitution, zweitens als Verband religiöser Organisationen (mit unterschiedlichen Aufgabenund Dienstleistungsbereichen), und drittens als Kirchengemeinde vor Ort.

Die Kommunikation der Kirche als Glaubensinstitution ist eine theologische Frage, die hier sicher nicht mit der genügenden Gründlichkeit behandelt werden kann. Mit dem schlichten und richtigen Hinweis, $\mathrm{da} B$ die Kirche schon immer für den Glauben "geworben" habe, ist leider nicht viel getan. Indessen erscheint mir das, was in der Missionswissenschaft unter dem Stichwort "Inkulturation" diskutiert wird, völlig kompatibel zu sein mit dem methodischen Ansatz des Social Marketing, nämlich Angebot und Nachfrage (in diesem Fall die Darstellung von Glaubensinhalten in unterschiedlichen Kulturkontexten betreffend) in bestmöglicher Weise zur Deckung zu bringen, ohne die Qualität der eigenen Leistung in irgendeiner Weise zu korrumpieren, sondern - im Gegenteil - sie gerade dadurch stetig zu verbessern.

Was die Kommunikation der Kirche als Organisation betrifft bzw. die Kommunikation all jener Organisationen, die für die Kirche stehen (angefangen bei den Generalvikariaten bis hin zum kirchlichen Krankenhaus), so steht die Notwendigkeit außer Frage, das gesamte Spektrum der Auftragskommunikation - von der Marketingkonzeption bis hin zur bezahlten Werbung - in professioneller Weise zu nutzen. Einrichtungen der Kirche, die als Unternehmen im Wettbewerb stehen, wird das in noch stärkerem Maße betreffen als Verwaltungs- und Dienstleistungsorganisationen, die auf gemeinnütziger Basis im Erziehungswesen, in der Krankenpflege oder anderswo karitativ tätig sind. Letztere werden die Mittel der Werbung weniger nutzen, sind aber wie Behörden, staatliche Einrichtungen und gemeinnützige Organisatio-

30 So lautet denn eine weitere mögliche Lehre aus dem kürzlich ergangenen Benetton-Urteil (vgl. Anm. 14): Die Werbung kann nicht einerseits die Rechte des Journalismus in Anspruch nehmen, ohne sich andererseits den Pflichten zu beugen. Oder anders herum formuliert: Wenn man der Werbung einerseits verbietet, das Leid in der Welt zu thematisieren, wird man es ihr bei anderer Gelegenheit nicht gebieten können. 
nen anderer Träger - auf professionelle Öffentlichkeitsarbeit zunehmend angewiesen. ${ }^{31}$

Die Kirchengemeinden schließlich werden nur in Ausnahmefällen über die finanziellen und personellen Ressourcen verfügen, um professionelle Öffentlichkeitsarbeit zu betreiben oder Werbeflächen zu bezahlen. Das eine oder andere läßt sich möglicherweise durch zahlungskräftige Gemeindemitglieder ausgleichen, die außer für den Kirchenbau beispielsweise auch einmal für den Druck von Plakaten oder T-Shirts für die Gemeindejugend aufkommen könnten. Auch die Zusammenarbeit mit Sponsoren ist denkbar, wobei eine hinreichende thematische Affinität zwischen den Beteiligten gegeben sein muß, um einen positiven Imagetransfer zu erreichen und ungünstige Ausstrahlungseffekte $\mathrm{zu}$ vermeiden. Insgesamt sind es jedoch vor allem die begrifflichen und strategischen Instrumente von Social Marketing, Öffentlichkeitsarbeit und Werbung, die für die Gemeindepastoral auch ohne den Anspruch auf Professionalität ausgewertet und fruchtbar gemacht werden können. ${ }^{32}$

Trotz der hier vorgenommenen Differenzierung ist zu beachten, daß der öffentliche Auftritt kirchlicher Einrichtungen - gleich ob als Institution, Organisation oder Gemeinde - immer auch das Gesamtbild der Kirche in der Öffentlichkeit prägt und seinerseits von diesem Gesamtbild nicht unabhängig ist. Außerdem besteht eine Besonderheit vieler kirchlicher Einrichtungen darin, sich einerseits als professionell arbeitende Organisation, andererseits als Teil des Gemeindelebens zu verstehen, wie z.B. die "Caritas", die daraus einen Leistungs- und Glaubwürdigkeitsvorteil für ihre Arbeit ableitet. In jedem Einzelfall ist deshalb zu überlegen, inwieweit die Entscheidung für oder gegen die Nutzung der Auftragskommunikation die Kirche nicht nur in ihrer jeweiligen Erscheinungsform, sondern als ganze betrifft. Das soll nicht heißen, daß beispielsweise ein im Wettbewerb stehendes Finanzdienstleistungsunternehmen der Kirche keine professionelle Werbung für günstige Kredite machen darf, sondern nur, daß es in einer besonderen Verantwortung steht, seine Kunden nicht in die Überschuldung zu treiben, und darauf auch im Stil der Werbung achten muß.

Wie stellen sich nach diesen Überlegungen nun mögliche Antworten auf unsere Ausgangsfragen dar?

31 Ein überzeugendes Beispiel für die auf einer Marketing-Konzeption aufbauende Öffentlichkeitsarbeit und Werbung eines katholischen Krankenhauses wird vorgestellt von Klaus Elfes, „Der Patient im Mittelpunkt. Modernes Dienstleistungsmarketing für das "Produkt' Krankenhaus", in: Praxis des Social Marketing, Michael Krzeminski/Clemens Neck (Hg.), Frankfurt/M. Institut für Medienentwicklung und Kommunikation 1994, 37-60.

32 Vgl. Bernhard Langenstein (Bearb.), Öffentlichkeitsarbeit. Gemeinden werben für den Glauben, Themenhefte Gemeindearbeit 8, Aachen: Bergmoser + Höller 1991, sowie G. Fauconnier, "Kerk, media en marketing", in: Openbaring en openbaarheid. Relaties tussen media en levensbeschouwing, Nijmegen: Katholiek Instituut voor Massamedia/Arbar 1989, 57-76. 
Erstens zur Frage, ob sich die Kirche auch bezahlter Kommunikationsdienstleistungen und solcher Kommunikationsmittel bedienen darf, die üblicherweise zu kommerziellen Zwecken genutzt werden. Nach der hier vorgenommenen Analyse sollten keine Bedenken bestehen, Aufgaben der Öffentlichkeitsarbeit von darauf spezialisierten Unternehmen (Berater, Agenturen) ausführen zu lassen und bei Bedarf auch bezahlte Werbeträger (Außenwerbeflächen, Anzeigenraum, Sendezeit) in Anspruch zu nehmen. Der Funktionszuwachs und die Intensivierung der öffentlichkeitsgerichteten Kommunikation erfordern eine zunehmende Professionalisierung gesellschaftsbezogener Kommunikationsangebote und fördern damit die Entstehung gewerblicher Kommunikationsdienstleister. Problematisch ist nicht, Offentlichkeitsarbeit und Werbung als gewerbliche Dienstleistung bzw. Infrastruktur in Anspruch zu nehmen, sondern allenfalls, daß diese in Stil und Inhalt dem Gesamterscheinungsbild und dem besonderen Kommunikationsauftrag der Kirche entsprechen müssen. Daher genügt es beispielsweise nicht, mittels einer Anzeigenkampagne oder Plakatwerbung "irgendwie" Aufmerksamkeit zu erzielen. Form und Thema der Aussagen sollten sich nicht primär an werbeästhetischen Gesichtspunkten orientieren, sondern auf einem umfassenden Kommunikationskonzept beruhen, wie es beispielsweise mit den Methoden des Social Marketing entwickelt werden kann.

Auch die zweite unserer Ausgangsfragen erscheint damit einer Beantwortung näher: Nicht die Delegation von Kommunikationsaufgaben an sich sollte ein Problem darstellen, sondern die Art der Zusammenarbeit zwischen den Stellen, die Verantwortung für die Außendarstellung der Kirche tragen, und solchen, die im Hause oder extern hochspezialisierte Kommunikationsdienstleistungen in Öffentlichkeitsarbeit oder Werbung erbringen. Auch hier muß Einigkeit über den Stil und das Gesamterscheinungsbild des öffentlichen Auftritts im Sinne einer Corporate Identity oder eines Organisationsleitbildes herrschen, und Loyalität sowie Legitimität müssen durch möglichst breite Mitwirkungsmöglichkeiten an der Erstellung dieses Leitbildes gesichert werden. Zu vermeiden ist die Zusammenarbeit mit Agenturen, die nicht bereit sind, diesen internen Meinungsbildungsproze $B$ über Stil und Inhalt des öffentlichen Auftritts mitzugestalten und stattdessen vorschlagen, ein solches Konzept von außen und nur der eigenen professionellen Logik folgend zu entwickeln.

Die dritte Frage, ob die mit der Nutzung der Auftragskommunikation verbundene Professionalisierung nicht die Authentizität und Glaubwürdigkeit kirchlicher Botschaften gefährde, ist vielleicht die schwierigste. Im Rückgriff auf ein bekanntes Axiom der Kommunikationswissenschaft, nämlich $\mathrm{da} B$ man nicht nicht kommunizieren kann $^{33}$, möchte ich sie hier versuchsweise wie folgt beantworten: Ohne die Werkzeuge professioneller Kommunikationsarbeit sind Glaub-

33 Vgl. Paul Watzlawick et al., Menschliche Kommunikation. Formen, Störungen, Paradoxien, Bern: Huber 1969. 
würdigkeit und Authentizität in der Öffentlichkeit gar nicht zu vermitteln! Das gilt übrigens nicht erst im Zeitalter der neuen Medien: Mahatma Gandhi und Martin Luther King waren nicht nur glaubwürdige Menschen, sondern sie haben sich und ihr Anliegen auch glaubwürdig in Szene gesetzt. ${ }^{34}$ Wenn heute ein gesellschaftspolitischer Kongreß ohne direkte Publikumsbeteiligung nur vor Pressevertretern und den Kameras der Fernsehanstalten stattfindet, ist die Veranstaltung nicht schon deshalb weniger glaubwürdig, weil man sie für die Medien inszeniert. Authentizität und Glaubwürdigkeit stehen also nicht im Gegensatz zur Inszenierung, sondern werden durch sie in der Öffentlichkeit erzeugt. Womöglich kann man öffentliche Ereignisse gar nicht nicht inszenieren, man kann sich nur bemühen, dies gut und nicht schlecht zu tun.

SUMMARY: Commissioned Communication for Church: Advertising, Public Relations and Social Marketing

It can be expected that the current changes in the media system of the industrialized societies are to result in a further growing importance of commissioned communication in its various forms. The aim of this article is to define the possible contribution of advertising, public relations and social marketing for communication tasks of the Church as well as to plea for their appropriate representation within the overall spectrum of Church communication planning. For this purpose the above mentioned fields of commissioned communication are described in more detail and clearly separated from journalistic and artistic forms of media production. While looking at the specific communication aims of the Church as instituion, organisation and community, it is finally possible to answer the following questions: Is it adviseable to make use of commissioned communication for the Church? How far can communication tasks of the Church be delegated at all? Does professionalization of Church communication not jeopardize its credibility and authenticity?

RÉSUMÉ: Communication commissionnée pour l'église: Publicité, PR et Social Marketing

Les changements permanents dans le système des médias des sociétés industrialisées indiquent que l'importance des differentes formes de la communication commissionee continuent à grandir. Le but de cet article c'est de définir la possible contribution de publicité, PR et Social Marketing aux tåches de communication de l'église et d'intercéder pour sa considération dans le système total de la planification de la communication de l'église. A cet effet on présentera en détail les champs d'activités mencionnés de la communication commissionée et on les différencie clairement des formes journalistiques et artistiques de la production des medias. Si se considère les différentes táches specifiquement social-communicatives de l'église comme institution, organisati-

34 Vgl. Keval J. Kumar, "Gandhian Techniques of Mass Communication", in: Communicatio Socialis Yearbook V, Indore: Satprakashan Sanchar Press 1986, 61-76. 
on et communauté, il es possible de répondre aux questions suivantes: Peut-on appliquer les moyens de la communication commissionnée pour les buts de communication de l'église? Est-ce possible de déléguer les tâches de communication? Est-ce que la communication de l'église perd sa crédibilité y sa authenticité par suite de sa professionalisation?

RESUMEN: Comunicación comisionada para la Iglesia: Publicidad, PR y Social Marketing

Considerando los cambios continuos en el sistema de los medios de comunicación de las sociedades industrializadas, es de esperar que la importancia de las diferentes formas de la comunicación comisionada sigue creciendo. El objeto de este artículo es definir la posible contribución de la publicidad, PR y Social Marketing a las tareas de comunicación de la Iglesia y abogar por su consideración adecuada en el conjunto del planeamiento de comunicación de la Iglesia. Con este fin se presentarán más en detalle los campos de acción arriba mencionados de la comunicación comisionada para además diferenciarlos claramente de las formas periodísticas y artísticas de la producción de los medios de comunicación. Si se examinan las tareas específicamente social-comunicativas de la Iglesia como institución, organzación y comunidad se puede responder a la preguntas seguientes: ¿Se puede aplicar los medios de la comunicación comisionada para los fines de comunicación de la Iglesia? ¿Es realmente posible delegar tareas de comunicación? ¿No es así que la comunicación de la Iglesia pierde su credibilidad y su authenticidad profesionándola? 